\title{
A Deep Learning-Aided Detection Method for FTN-Based NOMA
}

\author{
Jianxiong Pan, Neng Ye $\mathbb{D}$, Aihua Wang $\mathbb{D}$, and Xiangming Li $\mathbb{D}$ \\ School of Information and Electronics, Beijing Institute of Technology, Beijing 100081, China \\ Correspondence should be addressed to Aihua Wang; wah@bit.edu.cn
}

Received 6 April 2019; Revised 4 June 2019; Accepted 5 September 2019; Published 29 January 2020

Guest Editor: Shuai Han

Copyright (C) 2020 Jianxiong Pan et al. This is an open access article distributed under the Creative Commons Attribution License, which permits unrestricted use, distribution, and reproduction in any medium, provided the original work is properly cited.

\begin{abstract}
The rapid booming of future smart city applications and Internet of things (IoT) has raised higher demands on the nextgeneration radio access technologies with respect to connection density, spectral efficiency (SE), transmission accuracy, and detection latency. Recently, faster-than-Nyquist (FTN) and nonorthogonal multiple access (NOMA) have been regarded as promising technologies to achieve higher SE and massive connections, respectively. In this paper, we aim to exploit the joint benefits of FTN and NOMA by superimposing multiple FTN-based transmission signals on the same physical recourses. Considering the complicated intra- and interuser interferences introduced by the proposed transmission scheme, the conventional detection methods suffer from high computational complexity. To this end, we develop a novel sliding-window detection method by incorporating the state-of-the-art deep learning (DL) technology. The data-driven offline training is first applied to derive a near-optimal receiver for FTN-based NOMA, which is deployed online to achieve high detection accuracy as well as low latency. Monte Carlo simulation results validate that the proposed detector achieves higher detection accuracy than minimum mean squared error-frequency domain equalization (MMSE-FDE) and can even approach the performance of the maximum likelihood-based receiver with greatly reduced computational complexity, which is suitable for IoT applications in smart city with low latency and high reliability requirements.
\end{abstract}

\section{Introduction}

With the rapid development of $5 \mathrm{G}$, higher demands have been brought forward for communication systems. The typical usage scenarios, such as smart city, virtual reality (VR), wearable computing, and the transmission of big data [1], will be effectively realized with the support of advanced radio access technologies in 5G. Smart city is a promising scenario which represents a whole new way of production and lifestyle characterized by automation and intelligence. Meanwhile, Internet of things (IoT), which enables humancomputer interaction and machine-to-machine (M2M) communications, will be the foundation of the services in smart city $[1,2]$. It is estimated that over 50 billion devices will be connected to the internet by 2020 . With IoT, numerous devices in future smart city can be closely linked and a more intelligent life will be expected. To this end, the spectral efficiency and transmission latency shall be greatly improved and reduced, respectively, under massive connected devices. To fulfill these requirements, novel radio access technologies are required, such as novel multiple access (MA) technologies, network architectures, encoding, and modulation methods [3-5].

Faster-than-Nyquist (FTN) is a promising modulation method to achieve high spectral efficiency [6]. In conventional digital communication theory, Nyquist points out that the symbol transmission rates must satisfy the Nyquist criterion in order to enable transmission without intersymbol interference (ISI). However, in 1975, Mazo [6] found that in band-limited additive white Gaussian noise channel, the normalized minimum Euclidean distance does not decrease when the symbol rate exceeds within $25 \%$ of the Nyquist rate. FTN signaling can boost up a transmission rate without consuming more bandwidth or increasing the number of transceivers' antennas. The process of FTN shaping destroys the orthogonality among symbols and introduces ISI unavoidably. In 1995, the authors in [7] proposed an achievable FTN transmission method, which uses the iterative method to design the filter. Due to the fact that the sinc pulse is unachievable in practical, the authors in 
[8] studied the raised cosine-like pulse and thoroughly analyzed the error patterns at different FTN rates. The usage of constraint coding may further increase the rate of the FTN system at a cost of about $1 \mathrm{~dB}$ power and complexity. Recently, FTN has received much more attention owing to high spectral efficiency requirement of 5G. The authors of [9] found the FTN pulses aliased at the same time in the time domain and the frequency domain. Also, the time and frequency domain intervals satisfying the minimum distance are searched. The simulation results of Anderson et al. [10] reveal that when the transmitted pulse has excessive bandwidth, FTN transmission can achieve higher capacity than transmission under Nyquist rate. In other works, the research of FTN has been extended on multicarrier systems $[11,12]$ and low-complexity demodulation [13].

However, most research studies about FTN only focus on the point-to-point communication, which contradicts to the massive connection requirement in future smart city and IoT. To enable a practical multiuser communication system, efficient resource mapping patterns [14] and new multiple access methods are required. In 5G communication, nonorthogonal multiple access (NOMA) has been recognized as the promising access protocol due to the superior performance in spectral efficiency, connectivity, and flexibility [15]. Nonorthogonal schemes allow overlapping among the signals from different users by exploiting power domain, code domain or interleaver pattern, etc., and thus can provide better performance than the orthogonal counterparts [16]. In NOMA, multiple users share the same physical resource in downlink to increase the system capacity. Successive interference cancellation (SIC) is usually utilized to distinguish signals from multiple users [17]. Recently, many research studies combine grant-free transmission with NOMA scheme to simultaneously accomplish high throughput, large connectivity, and low energy cost [18-20]. FTN transmission is also considered in NOMA scenario to gain superior performance in spectral efficiency [21, 22]. However, the gains are finite and the structure of the receiver is too complicated to be applied in low-latency scenes.

NOMA and FTN are all essentially nonorthogonal signal processing methods. The former is nonorthogonal between users and the latter is in the level of symbols. FTN destroys the orthogonality among symbols and introduces ISI unavoidably. In order to eliminate ISI and ensure accuracy, the detection algorithm at the receiver is usually extremely complicated. Some detection methods, e.g., Viterbi algorithm [8], minimum mean squared errorfrequency domain equalization (MMSE-FDE) [23], and Bahl-Cocke-Jelinek-Raviv (BCJR) detection [24], are studied to reduce the impact of ISI. The performance of Viterbi approaches maximum likelihood (ML) detection without ISI at the cost of huge complexity, which is obviously not suitable for the transmission scenarios with low latency requirements (e.g., VR and autopilot). Compared with Viterbi detections, FDE-based algorithm reduces the complexity to a certain extent but the usage of $\mathrm{CP}$ decreases the spectral efficiency and the detection accuracy does not meet the ideal requirement. Therefore, this paper aims to design a joint detection algorithm of
FTN-NOMA, where both low computational complexity and high detection accuracy are simultaneously achieved.

While Geoffrey Hinton rediscovered the potential of deep neural network (DNN) [25], deep learning (DL) has recently developed rapidly and has made huge achievements in various fields. It is especially skilled in solving the optimization problems for complex models in a data-driven fashion. Some previous research studies have considered the deployment of deep learning in wireless communication fields [26-29]. By utilizing deep networks, which has strong learning ability and universal approximation characteristic, DL can be deployed to simulate the communication system with complicated structure. Moreover, the data-driven optimization method can achieve the end-to-end optimization of the whole communication system as a whole. As a preliminary research, the authors in [4] exploited the joint benefit of grant-free access and NOMA transmissions to meet the requirements of tactile IoT with the aid of DL.

In this paper, we extend the typical FTN transmission into NOMA in multiuser scenario. To ensure the reliability and reduce detection latency, a DL-aided receiver method of FTN-NOMA named sliding window detection is proposed. First of all, we construct a neural network model of FTN's receiver to acquire the trained weight matrix and bias vector. Then, these matrixes are utilized to directly transform the received signals into transmitted bits with simple matrix multiplication and addition. The proposed scheme avoids the complicated iteration and convolution operations in conventional receivers, such as BCJR and Viterbi receiver. In addition, this scheme can ensure high detection accuracy which is close to the performance of ML.

The rest of this paper is organized as follows. The system model of multiuser FTN including the transmitter and receiver design is described in Section 2. In Section 3, we provide the specific proposed detection method based DNN. Simulation results are presented in Section 4, which validate the performance gain of the proposed detection scheme. Conclusions and future works are provided in Section 5.

\section{The Proposed FTN-NOMA System Model}

The paper considers an uplink two-user NOMA in AWGN channel. The system adopts the FTN method to transmit the modulated symbols with the shaping filter of root raised cosine (RRC) pulse, where ISI is introduced unavoidably. Considering the attenuation characteristic of RRC pulse, there exists little interference among symbols far apart. Therefore, we assume that each symbol is only interfered by two adjacent symbols, i.e., the number of detection nodes is equal to 3. Figure 1 describes our model of FTN transmission process in two-user case, in which the conventional demodulation receiver algorithms for FTN, e.g., Viterbi and FDE, are substituted by deep learning module.

2.1. Transmitter. For each user, after baseband modulation, the symbols would pass through the shaping filter $h(t)$. In the FTN method, the waveforms of each symbol are no longer orthogonal in time domain. The symbol time is 


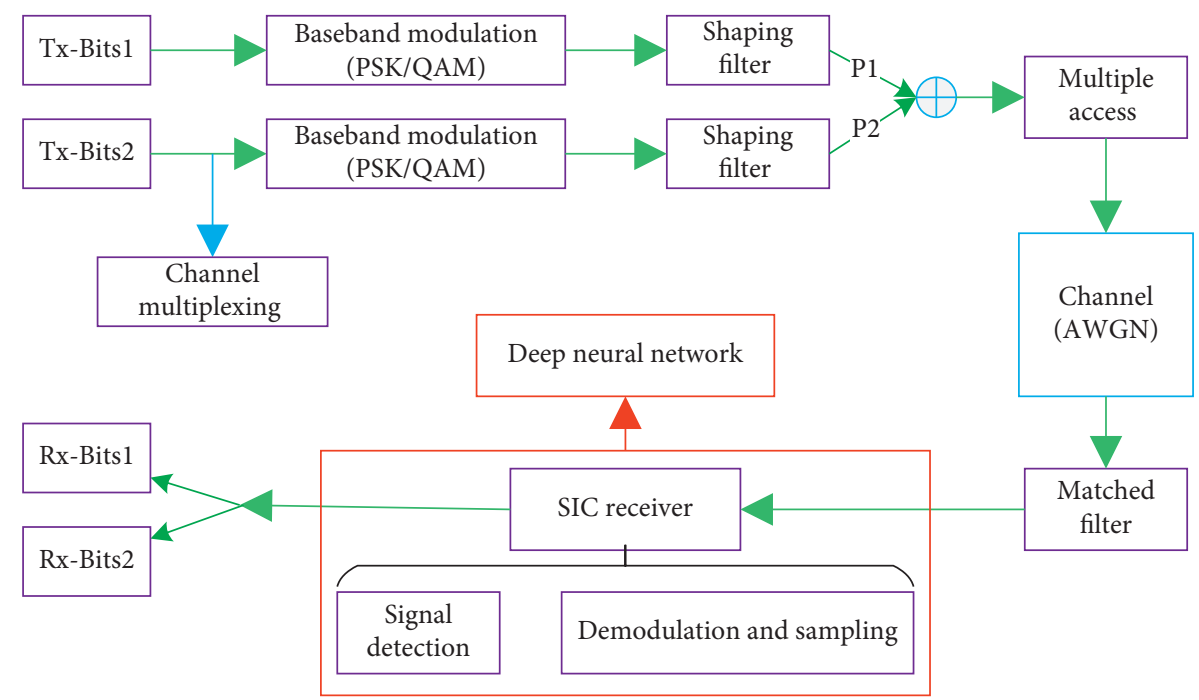

Figure 1: Transmission model of the proposed FTN-NOMA scheme.

shortened to $\alpha T, 0<\alpha<1$. Considering a point-to-point communication system, the general expression of singleuser FTN is

$$
s_{i}(t)=\sqrt{E_{s}} \sum_{n} a_{n} h(t-n \alpha T)
$$

where $E_{s}$ is the energy of BPSK symbols, $h(t)$ is the RRC shaping pulse with a roll-off factor $\beta=0.5, T$ is the symbol period satisfying Nyquist principle, $a_{n}$ is the $n$-th BPSK symbol, and $\alpha$ is the FTN compression factor. If $\alpha=1$, the whole process is equal to Nyquist transmission without any ISI. FTN reduces the distance among adjacent symbols in limited bandwidth and thus improves the spectral efficiency.

Figure 2 illustrates the transmitted symbols with Nyquist and FTN signaling, respectively. For each symbol's waveform, there exists the interference from other symbols, which is called ISI. The existence of ISI brings difficulty to the demodulation and detection of symbols.

In uplink NOMA case, the received waveforms at base station (BS) are the superposition of two users' FTN transmission symbols. The system model of uplink NOMA is shown in Figure 3, where there are two users in the cell and the signals from different users are superposed in power domain. Different from conventional multiple access methods, all users in the cell occupy the same time and frequency resources to transmit signals.

We assume that the two users are simultaneously multiplexed and user- 2 is in the center of the cell. So the transmission power of user-1 is smaller than user-2. The FTN superposition signal of two users can be described by

$$
x(t)=\sum_{i=1}^{2} \sqrt{P_{i}} s_{i}(t)=\sum_{i=1}^{2} \sqrt{P_{i}} \sum_{n} a_{n} h(t-n \alpha T),
$$

where $P_{i}$ represents the transmission power of user- $i$.

2.2. Receiver. Passing through AWGN channel, the received signal at BS becomes

$$
\begin{aligned}
& y_{k}=y(k \alpha T) \\
& =\sum_{i} h_{i} \times \sqrt{P_{i}} \sum_{n=1}^{N} a_{i, n} g(k \alpha T-n \alpha T)+\eta(k \alpha T) \\
& =\underbrace{h_{1} \times \sqrt{P_{1}} \times a_{k} g(0)}_{\text {Expected Signal }}+\underbrace{h_{1} \times \sqrt{P_{1}} \times \sum_{n \neq k} a_{n} g(k \alpha T-n \alpha T)}_{\text {ISI }} \\
& +\underbrace{h_{2} \times \sqrt{P_{2}} \sum_{n=1}^{N} a_{2, n} g(k \alpha T-n \alpha T)}_{\text {Interference among users }}+\underbrace{\eta(k \alpha T)}_{\text {Channel Noise }},
\end{aligned}
$$

where $h_{i}$ denotes channel response from user- $i$ to BS and additive white Gaussian noise is denoted by $\eta$. And $g(t)=$ $f(t) * f(t)$ is from the process of matched filtering.

Successive interference cancelation (SIC) is the general receiver algorithm of NOMA, which is performed at user- $i$ to eliminate the multiple access interference and improve SINR of the desired signal. The basic operation of SIC is shown in Figure 4. For the signal from user-2 (higher transmission power), there is no SIC processing. The base station directly conducts signal detection to detect the desired signal regarding the signal from user- 1 as noise. For user-1's signal, BS detects the signal from user-2 at first and then SIC is conducted to eliminate this interference. Finally, BS conducts signal detection to detect user-1's desired signal.

For single user, the process of signal detection is equal to the demodulation of FTN received symbols. In this paper, we consider two conventional detection algorithms, i.e., Viterbi decoding and FDE, to compare their performances with the proposed scheme.

2.2.1. Viterbi Algorithm. Viterbi algorithm is essentially the maximum likelihood (ML) detection method. However, different from the typical ML, Viterbi algorithm disperses the complexity into each symbol detection period. However, 


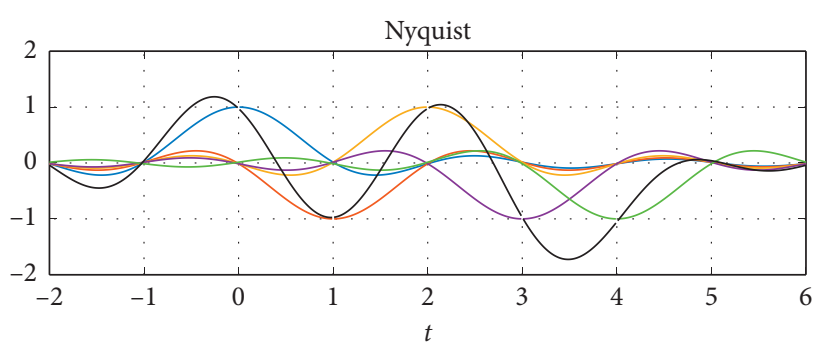

(a)

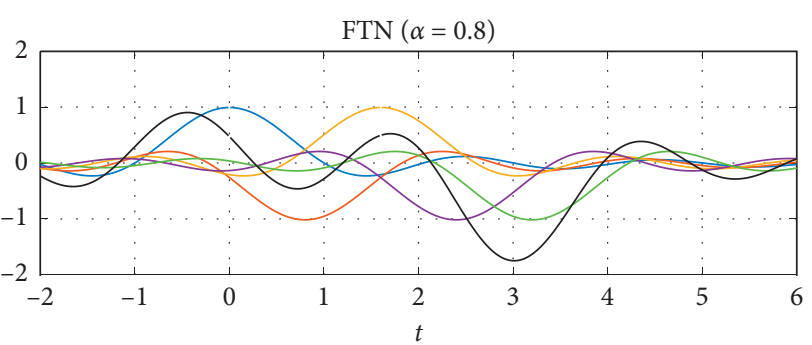

(b)

FIgure 2: Transmitted waveforms with Nyquist and FTN signaling.

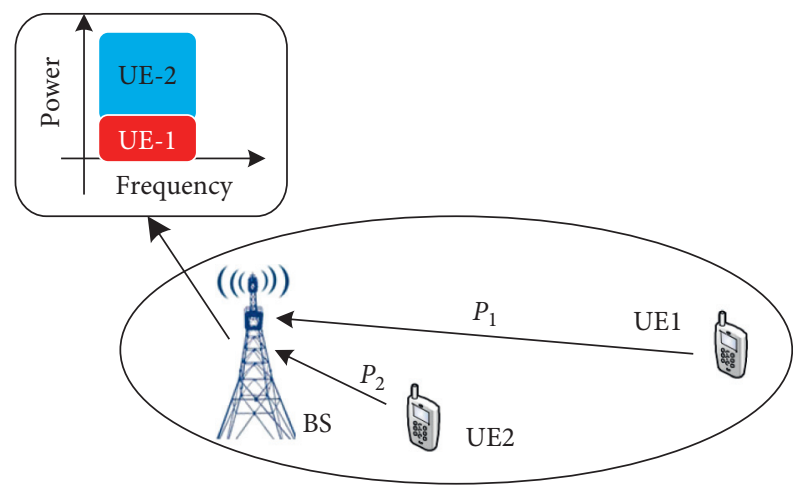

FIgURE 3: Uplink NOMA model in a cell with two users.

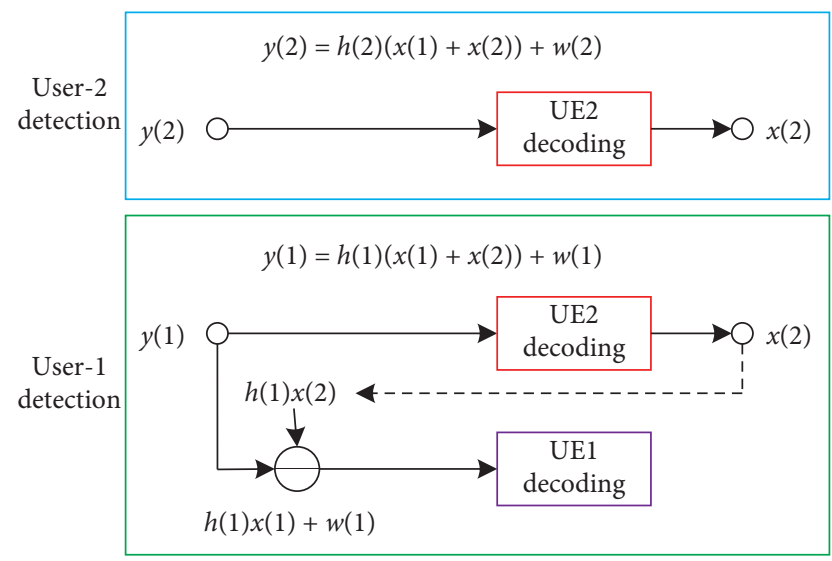

FIgURE 4: The detection process of the SIC receiver in two-user case.

the total complexity of Viterbi algorithm has no descent compared with the ML detection method. For FTN transmission, the Viterbi receiver has the optimum BER performance.

The process of FTN's Viterbi algorithm (BPSK modulation) is as follows:

(i) Assume that each FTN symbol is interfered by the adjacent two symbols. The Viterbi trellis, as demonstrated in Figure 5, shall contain 4 state nodes which are denoted as $a, b, c$, and $d$, respectively. Each state node represents two source bits:

$$
a-00 \quad b-01 \quad c-10 \quad d-11 .
$$

(ii) For each received symbol, list all possible combination of transmitted bits. Each node has two possible paths. For example (the underline parts represent the possible symbol/bit at this moment):

$$
\begin{array}{ll}
a: \ldots, 00 \underline{0}, \ldots & b: \ldots, 00 \underline{1}, \ldots \\
\ldots, 10 \underline{0}, \ldots & \ldots, 10 \underline{1}, \ldots \\
c: \ldots, 01 \underline{0}, \ldots & d: \ldots, 01 \underline{1}, \ldots \\
\ldots, 11 \underline{0}, \ldots & \ldots, 11 \underline{1}, \ldots
\end{array}
$$

(iii) Calculate the least Euclidean distance between all possible paths and the receive symbol at each moment:

$\min _{m} \int_{0}^{N T+\Delta}|y(t)-\widetilde{s}(t)|^{2} \mathrm{~d} t \quad m=1,2, \ldots, 8$,

where $y(t)$ is the received symbol and $\widetilde{s}(t)$ represents the possible path.

(iv) For each node, choose the path with smaller Euclidean distance from the two possible paths and note down its distance. Thus, 4 paths survive from the possible 8 paths. For example, the remaining paths are

$$
\begin{aligned}
& a: \ldots, 000, \ldots \quad b: \ldots, 101, \ldots \\
& c: \ldots, 110, \ldots \quad d: \ldots, 011, \ldots
\end{aligned}
$$

(v) Repeat the above process until the last received symbol is detected.

(vi) Choose the shortest one from the last 4 paths as the optimum detection result.

The whole process can be described by Figure 5 . It is worth mentioning that when the acceleration factor is no less than Mazo bound, the accuracy rate of Viterbi detection approaches the ML receiver without any ISI $(\alpha=1)$.

2.2.2. MMSE-FDE. FDE algorithm adds a short cyclic prefix into each transmission block to carry out fast Fourier transform- (FFT-) based low-complexity minimum-mean square error (MMSE) demodulation at the receiver. This 


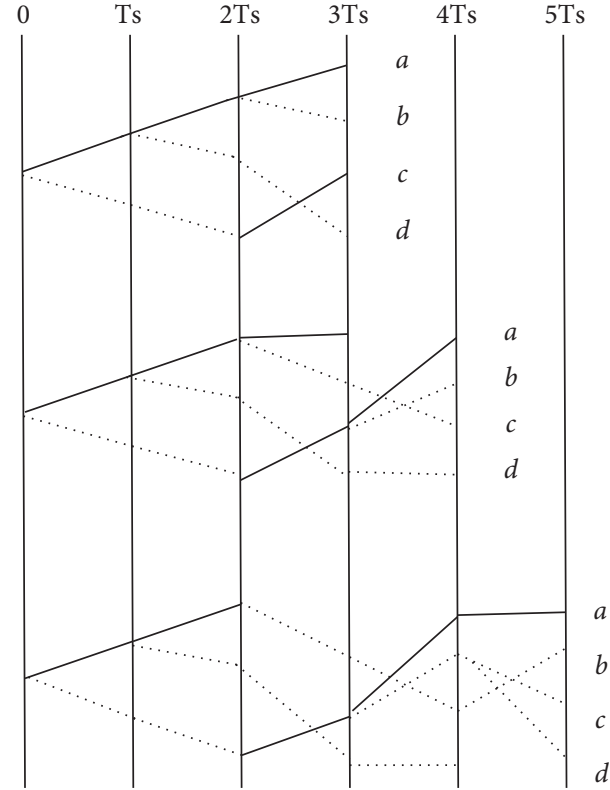

FIGURE 5: The process of Viterbi algorithm.

scheme is especially beneficial for a long-tap FTN case, where a delay spread associated with ISI is substantially large. The basic structure of FDE detection algorithm is illustrated in Figure 6.

At the transmitter, MMSE-FDE scheme divides the symbols into many blocks. Then, cycle prefix (CP) is added into the head of each block to reduce the interference among blocks. At the receiver, $\mathrm{CP}$ is first removed and then FFT transforms the received signal in time domain into frequency domain. ISI is eliminated by the process of frequency-domain equalization (FDE), the objective of which is to minimize the mean square error (MSE). Finally, the signal is transformed back into time domain by IFFT, and thus the interference among symbols is reduced. Compared with Viterbi algorithm, FDE can apparently reduce the complexity of detection process owing to the easy operation of FFT/IFFT. However, its accuracy rate is about $3 \sim 4 \mathrm{~dB}$ lower than the ML method. And the usage of CP reduces the spectral efficiency (SE) to a certain extent.

\section{DL-Aided Sliding Window Detection Scheme}

In this section, we will present the "sliding window detection" method based on deep learning. Figure 7 shows a set of consecutive symbols shaped by RRC pulses when the FTN acceleration factor is 0.8 and roll-off factor is 0.5 . As can be seen, for each received symbol, the greatest interference is from the pulses of two adjacent symbols while the interference from other symbols is not in the same order of magnitude as the adjacent symbols. So, the other interferences could be neglected. Based on this observation, we think that each received symbol stores the information of three transmitted bits (itself and two adjacent bits). In other words, every three received symbols can establish a mapping to a two-dimensional transmitted bit vector (in two-user case).
We can utilize deep neural network to model the mapping between three received symbols and one transmitted bit. Therefore, we create a symbol window whose size is three. As shown in Figure 8, the window moves by one symbol once until all received symbols are detected. Then, the complete mapping of all symbols is established. The whole process is like the sliding window so we name this scheme as "sliding window detection." At each sliding point, the real and imaginary parts of the three received symbols in the window are extracted. Thus, a matrix of six elements is formed as the input layer of the neural network:

$$
y=\left[\begin{array}{ccccc}
0 & y_{1, r} & y_{2, r} & \cdots & y_{N-1, r} \\
0 & y_{1, i} & y_{2, i} & \cdots & y_{N-1, i} \\
y_{1, r} & y_{2, r} & y_{3, r} & \cdots & y_{N, r} \\
y_{1, i} & y_{2, i} & y_{3, i} & \cdots & y_{N, i} \\
y_{2, r} & y_{3, r} & y_{4, r} & \cdots & 0 \\
y_{2, i} & y_{3, i} & y_{4, i} & \cdots & 0
\end{array}\right],
$$

and the output layer is a two-dimensional matrix including the transmitted bits from two users:

$$
x=\left[\begin{array}{lllll}
x_{1,1} & x_{1,2} & x_{1,3} & \cdots & x_{1, N} \\
x_{2,1} & x_{2,2} & x_{2,3} & \cdots & x_{2, N}
\end{array}\right] .
$$

The first transmitted bit vector $\overrightarrow{\mathbf{x}_{1}}$ and the last one $\overrightarrow{\mathbf{x}_{N}}$ map with only two received symbols. To keep the format of input layer, the head and tail of received symbol sequence are filled by zero. With the help of DNN, a mapping from the received symbol to the transmitted bit sequence is established. After a large amount of training steps, we can acquire the weight matrix $\mathbf{W}$ and bias matrix $\mathbf{B}$, which can be utilized to directly transform the received symbols into transmitted bits. In the condition of two-user case and BPSK modulation, the above is essentially equal to a four-category classification method.

The proposed network as shown in Figure 9 consists of input layer, output layer, and three hidden layers. Sigmoid function is used as activation function of the neural network, which can establish a nonlinear mapping for neurons. The expression of sigmoid function is

$$
f(x)=\frac{1}{1+e^{-x}} .
$$

In our proposed network, loss function is expressed by cross entropy. In the process of training steps, the loss function first maps the output values from the last layer to the interval of $(0,1)$ with sigmoid function and then calculates the cross entropy between the output value and training target:

$$
\operatorname{loss}_{i j}=-\left[x_{i j} * \ln p_{i j}+\left(1-x_{i j}\right) \ln \left(1-p_{i j}\right)\right],
$$

in which the expected output distribution is expressed by $x_{i j}$ while $p_{i j}$ represents the practical output distribution. In sigmoid function, the slope of the upper and lower boundary 


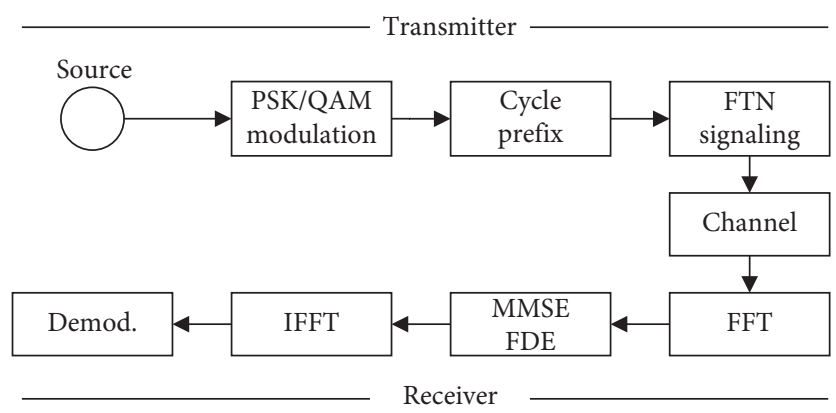

FIgURE 6: The process of MMSE-FDE algorithm.

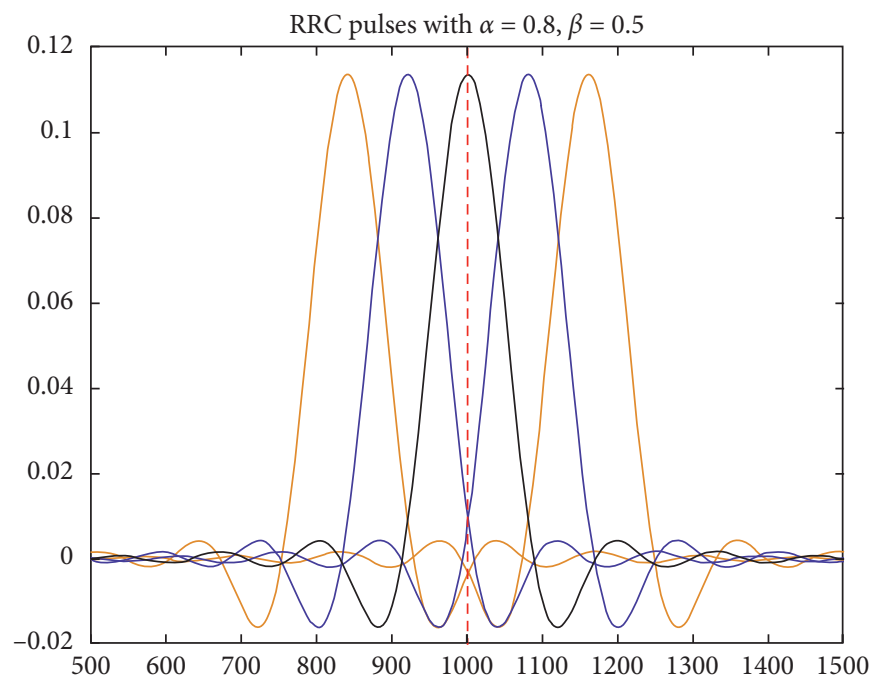

FIGURE 7: Superposition RRC pulses with FTN transmission.

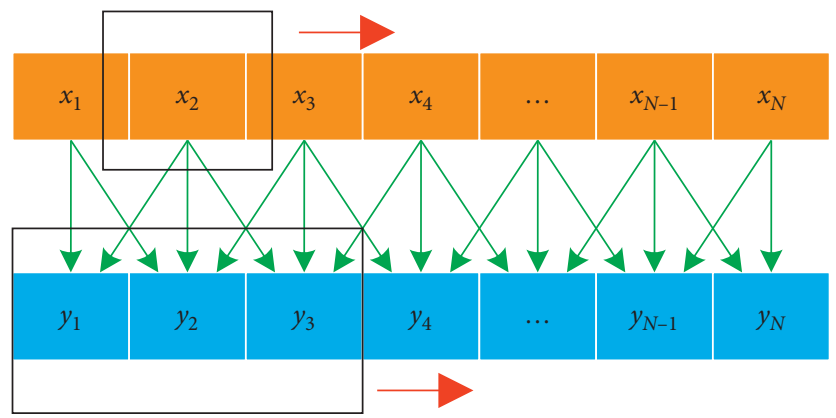

FIGURE 8: Illustration of the proposed sliding window detection method.

decreases rapidly; thus, the gradient value is extremely small when the training result is close to the real value, which slows down the convergence speed of the model. Cross entropy function is a logarithmic function which makes it maintain high gradient value when approaching the upper boundary. As a result, the convergence speed of the model will not be affected. So, the cross entropy can effectively show the similarity between the output value and the target distribution.

The objective of the training steps is to minimize the loss function. Here, we use adaptive moment estimation method (Adam) which is essentially a gradient descent method to optimize the parameter. The training steps of gradient descent are given in Algorithm 1. Different from the typical gradient descent method, Adam has special way to update the parameters. Assume that the random variable $X$ obeys a certain distribution; Adam constantly adjusts the learning rate $\alpha$ for each parameter according to the estimation of the first moment (i.e., the mean value of the sample) and the second moment (i.e., variance) of each parameter's gradient based on the loss function. Therefore, the learning speed of Adam can be controlled. In addition, the range of learning rate is limited, which can avoid big fluctuations of the network parameters, thus the value of the parameter in 


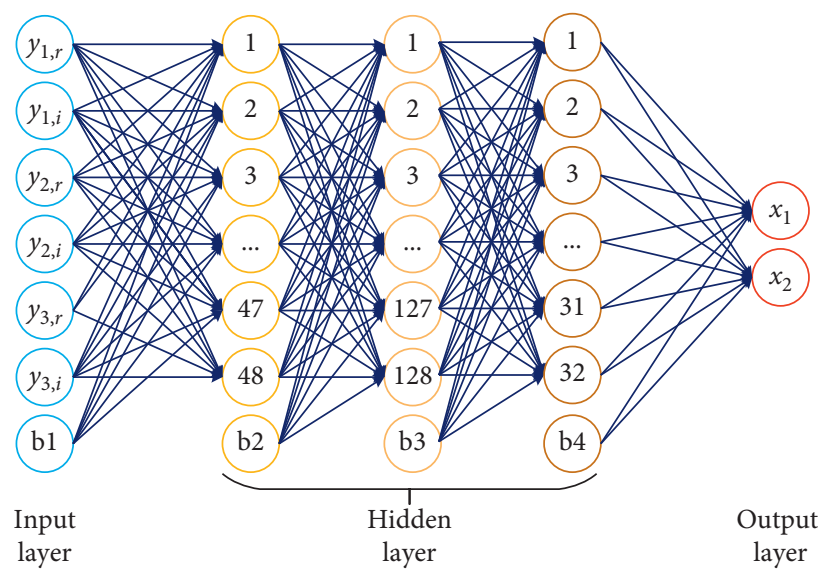

FIGURE 9: Structure of neural network for FTN-NOMA detection.

Input: $\mathbf{y}$ : training data; $\mathbf{x}$ : training labels; $\alpha$ : the learning rate;

Output: $\mathbf{W}^{(l)}$ : the connection weight matrix between layer $\mathbf{l}$ and layer $\mathbf{1}+1$

$\mathbf{b}^{(l)}$ : the bias matrix between layer $\mathbf{l}$ and layer $\mathbf{1}+1$

pred $^{(l)}$ : output of layer $l$

(1) Set $\Delta \mathbf{W}^{(l)}=0, \Delta \mathbf{b}^{(l)}=0$ (matrix/vector of zeros) for all $l$.

(2) For $i=1$ to $m$,

(a) Use backpropagation to compute the partial derivatives: $\nabla_{\mathbf{W}^{(l)}} \mathbf{J}(\mathbf{W}, \mathbf{b} ; \mathbf{y}, \mathbf{x})$ and $\nabla_{\mathbf{b}^{(l)}} \mathbf{J}(\mathbf{W}, \mathbf{b} ; \mathbf{y}, \mathbf{x})$.

(i) Perform a feedforward pass, computing the activations for layers 1 to $\mathbf{n}_{l}\left(\mathbf{n}_{l}=5\right)$ and using the equation defining the forward propagation steps.

(ii) For the layer $\mathbf{n}_{l}$, set $\boldsymbol{\delta}^{(l)}=\operatorname{pred}^{(l)}-\mathbf{y}^{(l)}$.

(iii) For the layer $\mathbf{l}=\mathbf{n}_{l}-1, \mathbf{n}_{l}-2, \ldots, 3,2$ set $\boldsymbol{\delta}^{(l)}=\left(\mathbf{W}^{(l)}\right)^{\mathrm{T}} \boldsymbol{\delta}^{(l)} \mathbf{f}^{\prime}\left(\mathbf{z}^{(l)}\right)$.

(iv) Compute the desired partial derivatives:

$\nabla_{\mathbf{W}^{(l)}} \mathbf{J}(\mathbf{W}, \mathbf{b} ; \mathbf{y}, \mathbf{x})=\boldsymbol{\delta}^{(l+1)}\left(\text { pred }^{(l)}\right)^{\mathrm{T}}$

$\nabla_{\mathbf{b}^{(l)}} \mathbf{J}(\mathbf{W}, \mathbf{b} ; \mathbf{y}, \mathbf{x})=\boldsymbol{\delta}^{(l+1)}$

(b) Set $\Delta \mathbf{W}^{(l)}=\Delta \mathbf{W}^{(l)}+\nabla_{\mathbf{W}^{(l)}} \mathbf{J}(\mathbf{W}, \mathbf{b} ; \mathbf{y}, \mathbf{x})$.

(c) Set $\Delta \mathbf{b}^{(l)}=\Delta \mathbf{b}^{(l)}+\nabla_{\mathbf{b}^{(l)}} \mathbf{J}(\mathbf{W}, \mathbf{b} ; \mathbf{y}, \mathbf{x})$.

(3) Update the parameters:

$\mathbf{W}^{(l)}=\mathbf{W}^{(l)}-\alpha\left[(1 / m) \Delta \mathbf{W}^{(l)}+\lambda \mathbf{W}^{(l)}\right]$.

$\mathbf{b}^{(l)}=\mathbf{b}^{(l)}-\boldsymbol{a}\left[(1 / m) \Delta \mathbf{b}^{(l)}\right]$.

Algorithm 1: Gradient descent training algorithm of neural network.

AdamOptimizer is relatively stable. Adam optimizer improves the performance of typical gradient descent and promotes the dynamic adjustment of hyperparametric.

\section{Simulation Results}

In this section, the performances of the proposed sliding window detection scheme based on DL are presented. We consider single-user and two-user uplink NOMA cases where the ratio of transmitter power is $0.9: 0.1$. The root raised cosine (RRC) pulse whose roll-off factors are $\beta=0.3$ and $\beta=0.5$ is employed for shaping filter $h(t)$. BPSK symbols are adopted which are conveyed over the additive Gaussian white noise (AWGN) channel. For the process of FTN shaping, we consider two acceleration factors of $\alpha=0.8$ and $\alpha=0.5$. In addition to the proposed scheme, two conventional receiver algorithms, i.e., MMSE-FDE and Viterbi, are simulated as the references.
The training network consists of input layer, three hidden layers, and output layer with 6, 48, 128, 32, and 2 neurons, respectively. The proposed network is constructed and trained based on Tensorflow framework. The module of loss function, i.e., cross entropy in Tensorflow, is sigmoid_cross_entropy_with_logits, in which output values are mapped to the $(0,1)$ interval with sigmoid function at first, and then the cross entropy between the practical output value and training target is calculated. The AdamOptimizer module which can control the learning speed provided by Tensorflow environment is utilized to minimize the loss function.

In the following part of this section, we present the proposed scheme's performances of single-user and twouser cases.

4.1. Single-User FTN Case. For single-user case, the size of training set is $143360(14 * 1024 * 10)$. The input layer of the network which represents the received signals is a matrix of 


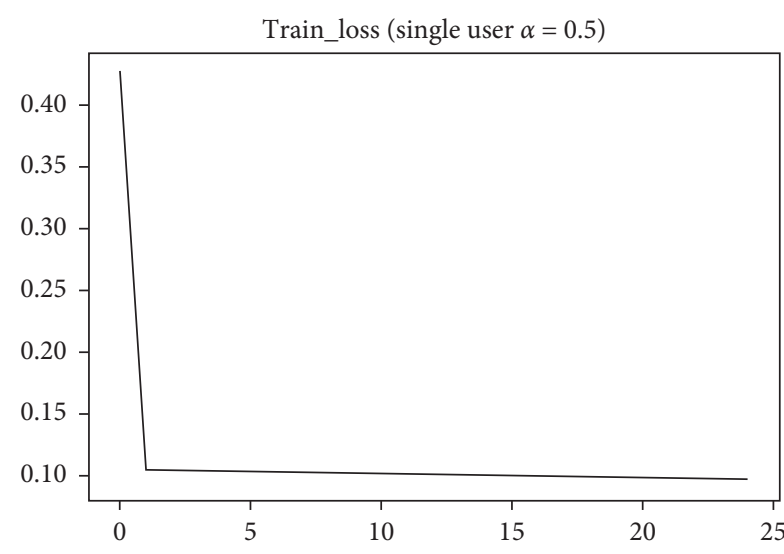

(a)

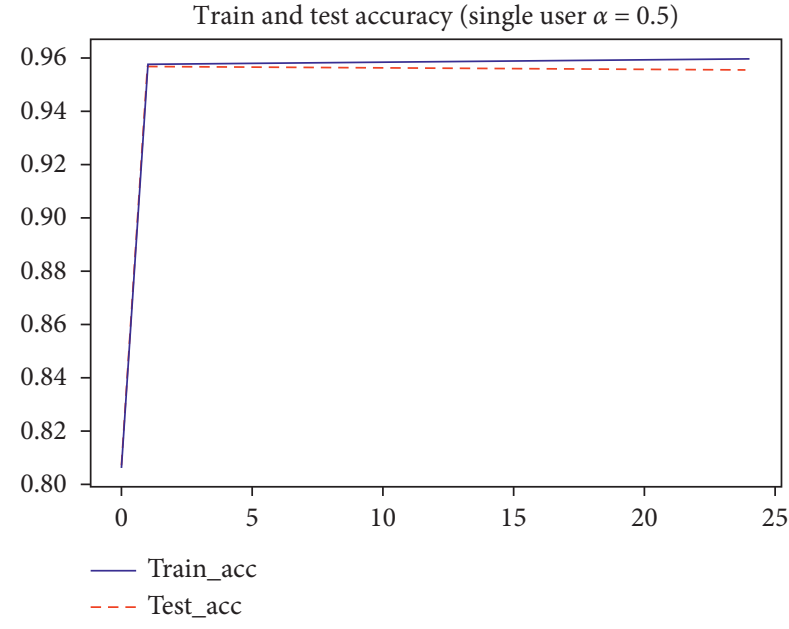

(b)

Figure 10: Training loss and accuracy versus training epoch. The unit of the $x$-coordinate is $10^{3}$ epochs. The DNN-based receiver is trained with $\alpha=0.5$ and $\beta=0.5$ in the single-user case.

$143360 * 6$ while the vector of output layer has 143360 elements. In addition, we generalize a test set which is the same size as training set to detect the accuracy of training steps. To prevent overfitting, we cut the training set into several blocks in the size of 100 . The learning rate of single user is set to 0.0001 . The number of the whole training step is 25000. To timely acquire the training effect, the network prints the loss values and accuracy rate of training set and test set per 1000 steps.

Figure 10 shows the trends of loss values and accuracy rate of $\alpha=0.5$. The total training steps are set to 25000. From the figure, we can find that the loss values and accuracy rate tend to be convergent after 10000 steps of training, where the convergence value of accuracy is about 0.96 , which approaches the accuracy rate of Viterbi detection algorithm when SNR is $4 \sim 6 \mathrm{~dB}$, i.e., the SNR point of dataset. The above results verify that DL-aided detection scheme is feasible to reach the ideal performance.

Figure 11 illustrates the BER performance of our proposed sliding window detection scheme compared with Viterbi and FDE when $\alpha=0.8$. Here, we adopt two RRC shaping filters of $\beta=0.3$ and $\beta=0.5$. For the FDE method, two kinds of CP lengths of 2 and 144 are considered. The baseband modulation scheme is BPSK.

As can be seen, our proposed scheme achieves a better performance than the FDE method. Especially when $\beta=0.5$, the proposed scheme can approach Viterbi algorithm with the gap of about only $1 \mathrm{~dB}$. As noted above, Viterbi algorithm can achieve the best BER performance of FTN at the cost of high complexity. Compared with the Viterbi receiver, our scheme has much lower computational complexity which can be obviously observed in the simulation process. Different from FDE, it does not use CP in AWGN channel and thus avoids the loss of spectral efficiency. Therefore, our proposed DL-aided scheme can make a good trade-off among the performances of detection accuracy, spectral efficiency, and computation complexity.
The BER performances of the above three schemes with $\alpha=0.5$ are illustrated in Figure 12. In this case, the accelerate factor $\alpha$ is lower than the Mazo limit and thus the BER performance of the FDE receiver has error floor. The Viterbi receiver can still achieve good detection performance at the cost of high computational complexity owing to the increase of state nodes. The BER performance of our proposed scheme can approach that of the Viterbi receiver while maintaining low computation complexity. Above results verify that our proposed scheme based on DL algorithm can combat strong ISI with low computational complexity in single-user case.

4.2. Multiuser Uplink FTN-NOMA Scene. In this section, we consider a two-user uplink FTN-NOMA case where the ratio of transmitter power is $0.9: 0.1$. To match the characteristics of two users simultaneously, the training set is collected from several signal to noise ratio (SNR) points. Considering the discontinuities of head and tail, we reduce the number of symbols. The size of training set is $84000(14 * 60 * 100)$. The input layer of the network which represents the received signals is a matrix of $84000 * 6$ while the vector of output layer has 84000 elements. In addition, we generalize a test set which is the same size as training set to detect the accuracy of training steps. In order to prevent overfitting, we cut the training set into several blocks with a size of 128 . The learning rate of two-user is set to 0.0005 . The number of the whole training step is 25000 .

The performances of two-user FTN-NOMA case are illustrated in Figure 13. The proposed scheme, i.e., sliding window detection, is compared with the conventional SIC receiver where each user's signals are demodulated with Viterbi algorithm. For the SIC receiver, it is clear that BER performance of the user with higher transmitter power approaches single-user scenario with the Viterbi receiver. And the performance of lower powered user is about $10 \mathrm{~dB}$ 


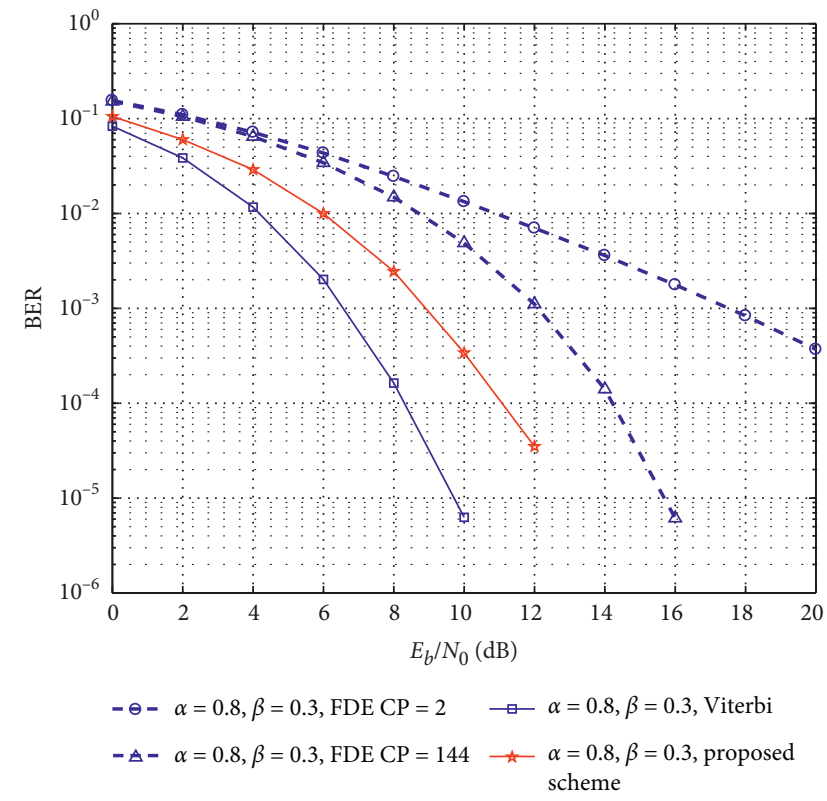

(a)

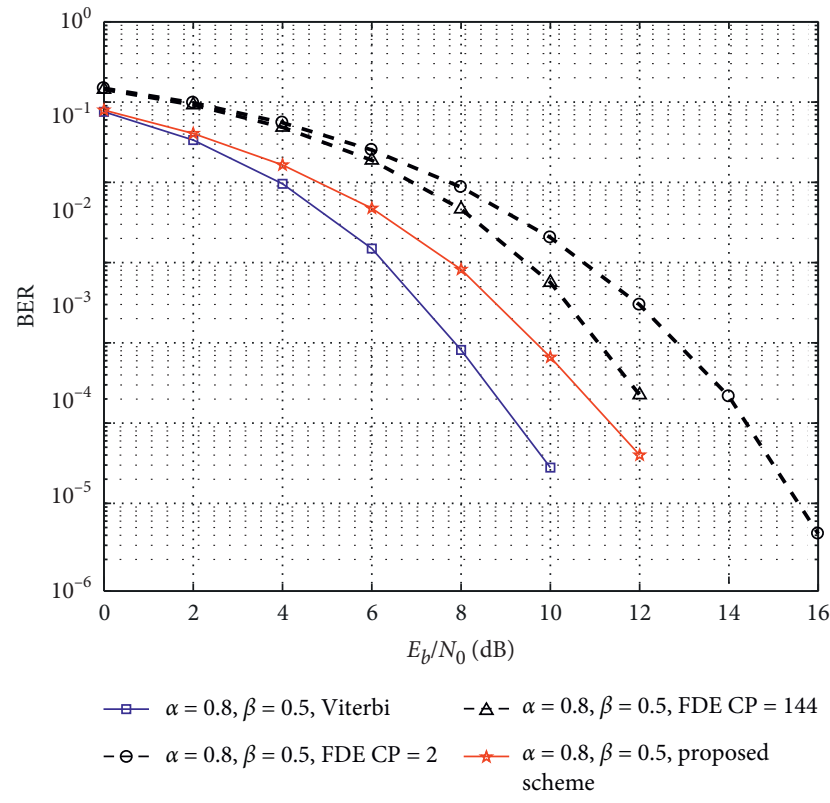

(b)

FIGURE 11: (a) BER performance of the proposed scheme, Viterbi, and FDE algorithms in single-user case at $\alpha=0.8$ and $\beta=0.3$. (b) BER performance of the proposed scheme, Viterbi, and FDE algorithms in single-user case at $\alpha=0.8$ and $\beta=0.5$.

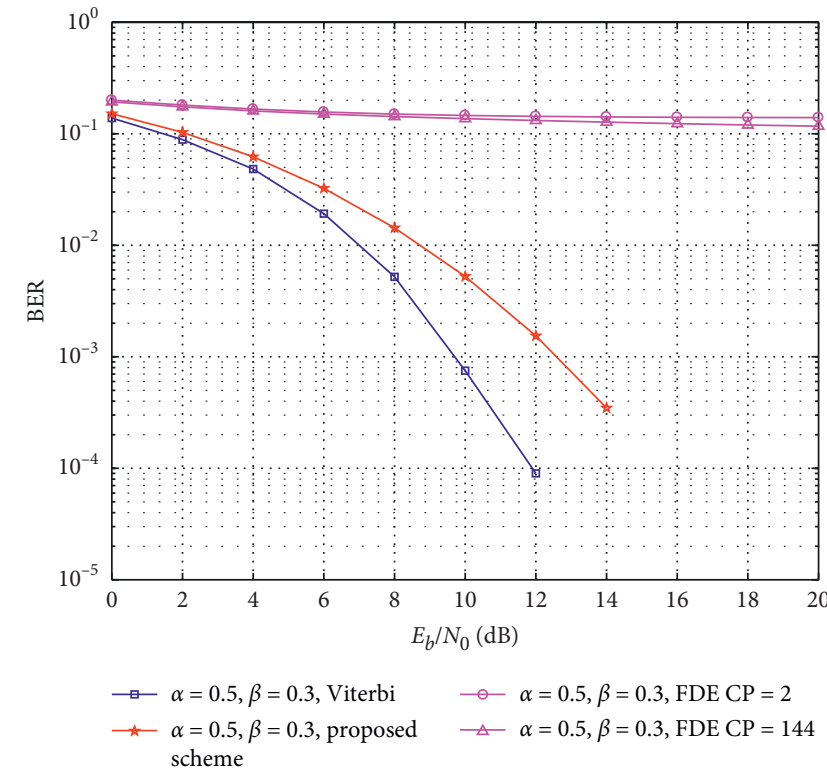

(a)

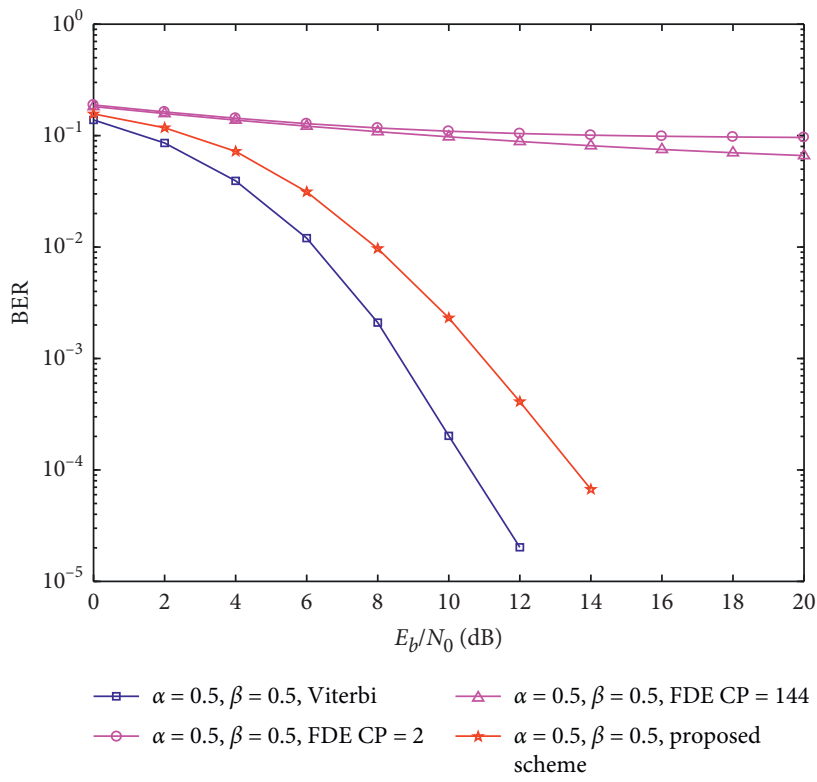

(b)

FIGURE 12: (a) BER performance of the proposed scheme, Viterbi, and FDE algorithms in single-user case at $\alpha=0.5$ and $\beta=0.3$. (b) BER performance of the proposed scheme, Viterbi, and FDE algorithms in single-user case at $\alpha=0.5$ and $\beta=0.5$.

worse than single-user case. For two-user FTN-NOMA case, the SIC receiver has double computational complexity than single-user scenario with the Viterbi detection method. As seen from Figure 12, the sliding window detection scheme has high detection accuracy which approaches the SIC receiver with Viterbi algorithm (ML detection method). In our proposed scheme, the descent of computational complexity is much more remarkable in NOMA case.
We assume that the training network consists of input layer, three hidden layers, and output layer with $a, b, c, d$, and $e$ neurons ( $e$ depends on the number of users). So, the sizes of four trained weight matrixes are $a \times b, b \times c, c \times d$, and $d \times e$ respectively. Our proposed scheme can directly transform the received symbols into transmitted bits with matrix multiplication operation and the computational complexity only depends on operation times of the matrix 


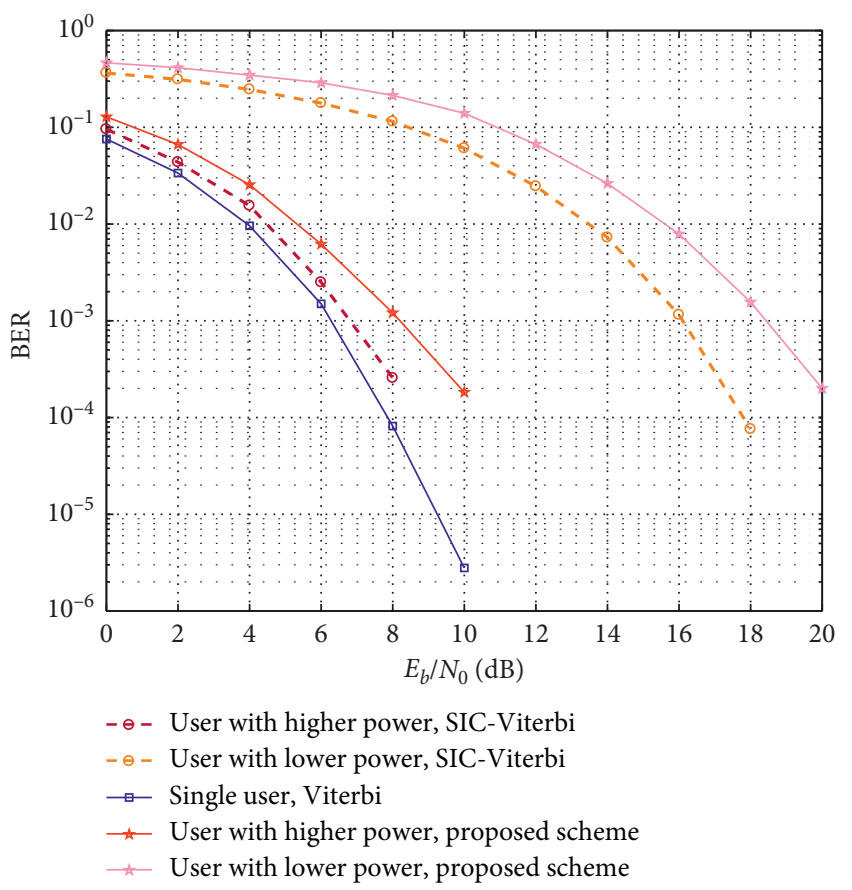

FIGURE 13: BER performance of the proposed scheme, Viterbi, and FDE algorithms in two-user case at $\alpha=0.8$ and $\beta=0.5$.

TABLE 1: Total multiplication operation times of different receiver schemes.

\begin{tabular}{lccc}
\hline & Viterbi & MMSE-FDE & Proposed scheme \\
\hline Single user & $N \times 2^{N}$ & $2 N^{2}+6 N$ & $N \times(a \times b+b \times c+c \times d+d)$ \\
$M$-user & $M \times N \times 2^{N}$ & $M \times\left(2 N^{2}+6 N\right)$ & $N \times(a \times b+b \times c+c \times d+d \times M)$ \\
\hline
\end{tabular}

elements. As mentioned above, the total complexity of the Viterbi receiver exponentially increases with the number of detected symbols. Considering $M$ users, $N$ transmitted bits of each user, and BPSK modulation, Table 1 provides the total times of multiplication operation with different receiver algorithms, which represents the computational complexity of these schemes. As can be seen, our proposed scheme has a remarkable effect on reducing the computational complexity of detection compared with the Viterbi receiver.

\section{Conclusions}

In this paper, we have extended the conventional FTN-based transmissions in NOMA and proposed a DL-aided receiver method of FTN-NOMA based on sliding window detection, which greatly reduces the computational complexity while maintaining high detection accuracy. Based on the datadriven optimization method, a neural network model of the FTN-NOMA receiver is constructed, which directly transforms the received signals into transmitted bits with simple matrix multiplication and addition. The proposed scheme avoids the complicated iteration and convolution operation of the conventional receiver like the BCJR or Viterbi method, which makes it suitable for low-latency scenes in smart city and IoT. Simulation results reveal that BER performance of our proposed scheme outperforms MMSE-
FDE algorithm and can approach the ML method which has the ideal performance in both OMA and NOMA cases. Moreover, the computational complexity can be remarkably reduced compared with the Viterbi or ML method due to the sliding window detection and DNN structure.

In the future, we will consider the DL-aided efficient detection methods of FTN-NOMA in multicarrier and multipath cases. In addition, we will study the robustness of DL methods considering high-order modulations in FTNNOMA.

\section{Data Availability}

No data were used to support this study.

\section{Conflicts of Interest}

The authors declare that there are no conflicts of interest regarding the publication of this paper.

\section{Authors' Contributions}

J. Pan and N. Ye contributed equally to this study.

\section{Acknowledgments}

This work was supported by the Advance Research Projects of 13th Five-Year Plan of Civil Aerospace Technology 
(B0105) and National Natural Science Foundation of China (61771051).

\section{References}

[1] S. Han, S. Xu, W. Meng, and L. Cheng, "An agile confidential transmission strategy combining big data driven cluster and OBF," IEEE Transactions on Vehicular Technology, vol. 66, no. 11, pp. 10259-10270, 2017.

[2] V. Gazis, M. Goertz, M. Huber et al., "IoT: challenges, projects, architectures," in Proceedings of the 2015 18th International Conference on Intelligence on Next Generation Networks, pp. 145-147, Paris, France, February 2015.

[3] S. Han, Yi Zhang, W. Meng, and H.-H. Chen, "Self-interference-cancelation-based SLNR precoding design for fullduplex relay-assisted system," IEEE Transactions on Vehicular Technology, vol. 67, no. 9, pp. 8249-8262, 2018.

[4] N. Ye, X. Li, H. Yu, A. Wang, W. Liu, and X. Hou, "Deep learning aided grant-free NOMA toward reliable low-latency access in tactile internet of Things," IEEE Transactions on Industrial Informatics, vol. 15, no. 5, pp. 2995-3005, 2019.

[5] S. Han, S. Xu, W. Meng, and Li Cheng, "Dense-device-enabled cooperative networks for efficient and secure transmission," IEEE Network, vol. 32, no. 2, pp. 100-106, 2018.

[6] J. E. Mazo, "Faster-than-Nyquist signaling," The Bell System Technical Journal, vol. 54, no. 8, pp. 1451-1462, 1975.

[7] C.-K. Wang and L.-S. Lee, "Practically realizable digital transmission significantly below the Nyquist bandwidth," IEEE Transactions on Communications, vol. 43, no. 2/3/4, pp. 166-169, 1995.

[8] A. D. Liveris and C. N. Georghiades, "Exploiting faster-thanNyquist signaling," IEEE Transactions on Communications, vol. 51, no. 9, pp. 1502-1511, 2003.

[9] F. Rusek and J. B. Anderson, "Constrained capacities for faster-than-nyquist signaling," IEEE Transactions on Information Theory, vol. 55, no. 2, pp. 764-775, 2009.

[10] J. B. Anderson, F. Rusek, and V. Owall, "Faster-than-nyquist signaling," Proceedings of the IEEE, vol. 101, no. 8, pp. 1817-1830, 2013.

[11] I. Kanaras, A. Chorti, M. R. D. Rodrigues, and I. Darwazeh, "Spectrally efficient FDM signals: bandwidth gain at the expense of receiver complexity," in Proceedings of the 2009 IEEE International Conference on Communications, pp. 1-6, Dresden, Germany, June 2009.

[12] S. Li, B. Bai, J. Zhou, Q. He, and Q. Li, "Superposition coded modulation based faster-than-nyquist signaling," Wireless Communications and Mobile Computing, vol. 2018, Article ID 4181626, 10 pages, 2018.

[13] P. Şen, T. Aktaş, and A. Ö. Yilmaz, "A low-complexity graphbased LMMSE receiver designed for colored noise induced by FTN-signaling," in Proceedings of the 2014 IEEE Wireless Communications and Networking Conference (WCNC), pp. 642-647, Istanbul, Turkey, April 2014.

[14] H. Yu, Z. Fei, N. Yang, and N. Ye, "Optimal design of resource element mapping for sparse spreading non-orthogonal multiple access," IEEE Wireless Communications Letters, vol. 7, no. 5, pp. 744-747, 2018.

[15] N. Ye, H. Han, L. Zhao, and A. Wang, "Uplink nonorthogonal multiple access technologies toward 5G: a survey," Wireless Communications and Mobile Computing, vol. 2018, Article ID 6187580, 26 pages, 2018.

[16] S. Han, Y. Huang, W. Meng, Li Cheng, N. Xu, and D. Chen, "Optimal power allocation for SCMA downlink systems based on maximum capacity," IEEE Transactions on Communications, vol. 67, no. 2, pp. 1480-1489, 2019.

[17] N. Ye, A. Wang, X. Li, W. Liu, X. Hou, and H. Yu, "On constellation rotation of NOMA with SIC receiver," IEEE Communications Letters, vol. 22, no. 3, pp. 514-517, 2018.

[18] N. Ye, A. Wang, X. Li, W. Liu, X. Hou, and H. Yu, "Rateadaptive multiple access for uplink grant-free transmission," Wireless Communications and Mobile Computing, vol. 2018, Article ID 8978207, 21 pages, 2018.

[19] K. Yang, N. Yang, N. Ye, M. Jia, Z. Gao, and R. Fan, "Nonorthogonal multiple access: achieving sustainable future radio access," IEEE Communications Magazine, vol. 57, no. 2, pp. 116-121, 2019.

[20] N. Ye, A. Wang, X. Li, H. Yu, A. Li, and H. Jiang, "A random non-orthogonal multiple access scheme for mMTC," in Proceedings of the 2017 IEEE 85th Vehicular Technology Conference (VTC Spring), pp. 1-6, Sydney, Australia, June 2017.

[21] A. T. Abebe and C. G. Kang, "Iterative decoders for FTNbased NOMA scheme to multiplex sporadic and broadband transmission," in Proceedings of the 2018 International Conference on Information and Communication Technology Convergence (ICTC), pp. 813-817, Jeju, Republic of Korea, October 2018.

[22] A. T. Abebe and C. G. Kang, "FTN-based MIMO transmission as a NOMA scheme for efficient coexistence of broadband and sporadic traffics," in Proceedings of the 2018 IEEE 87th Vehicular Technology Conference (VTC Spring), pp. 1-5, Porto, Portugal, June 2018.

[23] S. Sugiura, "Frequency-domain equalization of faster-thannyquist signaling," IEEE Wireless Communications Letters, vol. 2 , no. 5, pp. 555-558, 2013.

[24] L. Bahl, J. Cocke, F. Jelinek, and J. Raviv, "Optimal decoding of linear codes for minimizing symbol error rate (Corresp.)," IEEE Transactions on Information Theory, vol. 20, no. 2, pp. 284-287, 1974.

[25] G. E. Hinton and R. R. Salakhutdinov, "Reducing the dimensionality of data with neural networks," Science, vol. 313, no. 5786 , pp. 504-507, 2006.

[26] H. Li, "A neural network blind multi-user detection algorithm," in Proceedings of the 2009 International Conference on Measuring Technology and Mechatronics Automation, pp. 467-470, Zhangjiajie, China, April 2009.

[27] U. Mitra and H. V. Poor, "Neural network techniques for multi-user demodulation," in Proceedings of the IEEE International Conference on Neural Networks, vol. 3, pp. 15381543, San Francisco, CA, USA, March 1993.

[28] M. Ohta, "Neural networks for multiuser detector in asynchronous PC/SS communication system," in Proceedings of the Proceedings of the 2002 International Joint Conference on Neural Networks. IJCNN'02 (Cat. No.02CH37290), vol. 3, pp. 2809-2813, Honolulu, HI, USA, May 2002.

[29] F. Ibikunle and Y. X. Zhong, "Probabilistic neural networks for multi-user detection in code divisional multiple access communication channels," in Proceedings of the 1998 IEEE International Joint Conference On Neural Networks Proceedings. IEEE World Congress on Computational Intelligence (cat. No. 98CH36227), vol. 3, pp. 2557-2560, Anchorage, AK, USA, May 1998. 